\title{
Correction to: Is Open-Label Placebo a New Ergogenic Aid? A Commentary on Existing Studies and Guidelines for Future Research
}

\author{
Tiemi Saito ${ }^{1} \cdot$ Gabriel Barreto $^{1} \cdot$ Bryan Saunders ${ }^{1,2} \cdot$ Bruno Gualano $^{1,3}$
}

Published online: 29 April 2020

C) Springer Nature Switzerland AG 2020

\section{Correction to: Sports Medicine}

https://doi.org/10.1007/s40279-020-01285-w

Page 4, Figure 1:

"What factors meditate the response to open-label placebos?"

should read:

"What factors mediate the response to open-label placebos?"

The corrected figure has been copied below.

The original article can be found online at https://doi.org/10.1007/ s40279-020-01285-w.

Bruno Gualano

gualano@usp.br

1 Applied Physiology and Nutrition Research Group, School of Physical Education and Sport, Rheumatology Division, Faculdade de Medicina FMUSP, Universidade de Sao Paulo, Av. Dr. Arnaldo, 455, Cerqueira César, São Paulo, SP CEP: 01246903, Brazil

2 Institute of Orthopaedics and Traumatology, Faculty of Medicine FMUSP, University of São Paulo, São Paulo, Brazil

3 Food Research Center (FoRC), University of São Paulo, São Paulo, Brazil 
What we already know

Deceptive placebo treatments
elicit real responses
Expectation and pre-conditioning
may underlie the placebo effect
Open-placebos elicit genuine responses
in the clinical setting (e.g. cancer, irritable
bowel syndrome)
In sports science, open-label placebos
are underexplored, and existing
findings are conflicting

What is yet to be investigated

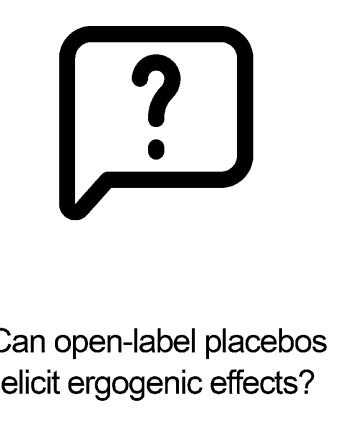

What factors mediate the response to open-label placebos?

(type of intervention, modality, training

status, sex, psychological profile, etc)

What are the physiological and biological mechanisms behind the effects of open-

$$
\text { label placebo? }
$$

What are the factors that could predict positive, negative or null responses to open-label placebo?

The original article has been corrected. 